\title{
Thermodynamic consistency, quark mass scaling, and properties of strange matter
}

\author{
C. J. Xia, ${ }^{1}$ G. X. Peng, ${ }^{1,2}$ S. W. Chen, ${ }^{1,3}$ Z. Y. Lu, ${ }^{1}$ and J. F. $\mathrm{Xu}^{1}$ \\ ${ }^{1}$ School of Physics, University of Chinese Academy of Sciences, Beijing 100049, China \\ ${ }^{2}$ Theoretical Physics Center for Science Facilities, Institute of High Energy Physics, Beijing 100049, China \\ ${ }^{3}$ China Information Technology Security Evaluation Center, Shangdixilu 8, Beijing 100085, China
}

\begin{abstract}
The previous thermodynamic treatment for models with density and/or temperature dependent quark masses is shown to be inconsistent with the requirement of fundamental thermodynamics. We therefore study a fully self-consistent one according to the fundamental differential equation of thermodynamics. After obtaining a new quark mass scaling with the inclusion of both confinement and leading-order perturbative interactions, we investigate properties of strange quark matter in the fully consistent thermodynamic treatment. It is found that the equation of state become stiffer, and accordingly, the maximum mass of strange stars is as large as about 2 times the solar mass, if strange quark matter is absolutely or metastable.
\end{abstract}

PACS numbers: 21.65.Qr, 05.70.Ce, 12.39.-x

\section{INTRODUCTION}

Since its possible absolute stability was conjectured nearly thirty years ago [1], strange quark matter (SQM) has been playing an important role in many interesting fields, for example, the deconfinement phase transition 2 [7], the hot and dense matter in heavy ion collisions 8], the structure of compact stars [9], etc. Lumps of SQM, the so-called strangelets [10], or slets [1], may exist in cosmic rays 12], and some of them might be on the way to our Earth [13, 14]. The neutron star could be converted to a quark star or mixed star due to leptonic weak interactions, or seeded with slets by the self-annihilating weakly interacting massive particles [15]. The structure of a strange quark star depends strongly on the stability of SQM which are still under active investigations [16. Presently, many aspects on quark matter are still left open. Among them the equation of state (EOS) is of special interest.

In principle, one has in hand the fundamental theory of strong interactions, i.e., quantum chromodynamics (QCD). Presently, however, no one can model quark matter exactly in QCD because of the known difficulty in the nonperturbative regime. The only case that can be exactly solved is the noninteracting system whose thermodynamic potential density $\Omega_{0}\left(T,\left\{\mu_{i}\right\},\left\{m_{i}\right\}\right)$ as a function of the temperature $T$, the chemical potentials $\left\{\mu_{i}\right\}$, and the particle masses $\left\{m_{i}\right\}$ can be found in many textbooks. For a free system, the particle mass is a constant, and the corresponding thermodynamic treatment is clearly known.

Nowadays, quark matter has been investigated with various phenomenological models, e.g., the Richard potential model [17, 18], the Nambu and Jona-Lasinio (NJL) model [19], the perturbation model [20], the field correlator method 21], the quark-cluster model 22], and many other models [23 28], etc. These models, to some extent, have a relation to, or start from, the freeparticle system. In the simplest version of the bag model [1, 10, 29], for example, a constant $B$, the so-called "bag constant", is added to the thermodynamic potential den- sity of the free system to reflect the quark confinement effect. This model has been applied in a vast number of investigations on the properties of SQM [30 33].

It is well known, however, that particle masses vary with medium. Such masses are usually called effective masses. In principle, not only masses will change but also the coupling constant will run in a medium [20]. The models with chemical-potential and/or temperature dependent particle masses are known as quasiparticle models 34], which have been explored in great detail over the past two decades 35 39]. A recent example is the model without density or temperature dependent infinity of the vacuum zero-point energy [40, 41].

Another important case is to include strong interquark interactions with density and/or temperature dependent quark masses. The original idea is to use a density dependent quark mass to express nonperturbative interaction effects [42, 43]. It was soon applied to study the equation of state (EOS) [44 51], the viscosity of SQM and dissipation of r-modes [52], the quark-diquark properties [53], and compact stars [54], etc. Until now, these kinds of models have been developed greatly [55 58].

The most disputable issues in these kinds of models is the thermodynamic inconsistency problem [44 49]. Let us take the zero-temperature case to explain the issue. Originally, all the thermodynamic formulas are taken as the same of a free system [44]. In this treatment (TD1), the properties of SQM are significantly different from these in the conventional bag model. Subsequently, an additional term was added to the pressure due to the density dependence of quark masses, and simultaneously, the additional term was subtracted from the energy density [45]. In this second treatment (TD-2), SQM can be self-bound. A serious problem is that the pressure at the minimum of the energy per baryon deviates obviously from zero. The third treatment (TD-3) has the additional term in the pressure to confine quarks, but it does not appear in the energy density [46]. TD-3 successfully overcomes the inconsistency between the zero pressure and energy minimum, and later extended to finite temperature [47]. 
To study the deconfinement phase transition, one needs to use true chemical potentials to maintain chemical equilibrium. It was shown [2] that the quark chemical potentials used in the original TD-3 are in fact effective ones. The true chemical potential $\mu_{i}$ of the quark flavor $i$ differs from the corresponding effective chemical potential $\mu_{i}^{*}$ by a common term for all quark flavors, and accordingly satisfies the same weak equilibrium conditions. For SQM, therefore, the effective chemical potentials act like the real chemical potentials to give the same EOS.

Recently, another effort has been made to clear the ambiguity in thermodynamic treatments, where the quark mass was regarded as an intrinsic freedom, and an additional term was added to the fundamental thermodynamic differential equation 48]. Assuming the effective mass intrinsic while it depends completely on the state variables (the density and/or temperature) is conceptually self-contradictory, and inevitably leads to inconsistency. In fact, with the additional term to the fundamental thermodynamic differential equation, the original TD-1 treatment was recovered. Because the pressure in this treatment is always positive, which poses a problem related to the stability of SQM, the authors finally had to consider the vacuum contribution by adding a term to the thermodynamic potential density, as had been done in a previous reference [49], to which we refer as TD-4.

Another important aspect very relevant to thermodynamic treatment is how the quark masses depend on the density and/or temperature. Originally, the density dependence of quark masses is parametrized as

$$
m_{i}=m_{i 0}+\frac{B}{3 n_{\mathrm{b}}},
$$

which was first given for light quarks in Ref. [42] according to bag model assumption, and extended to including strange quarks in Ref. [44]. An alternative parametrization of the density dependence is the cubic-root scaling

$$
m_{i}=m_{i 0}+\frac{D}{n_{\mathrm{b}}^{1 / 3}}
$$

derived from the linear confinement and leading-order inmedium chiral condensate [50]. In Eqs. (11) and (2), $m_{i 0}$ $(i=u, d, s)$ are the corresponding quark current mass, $n_{\mathrm{b}}$ is the baryon number density, $B$ and $D$ are constants signifying the confinement strength.

The purposes of the present paper are twofold. First, in the next section, we give the quantitative criteria for thermodynamic consistency, and compare the abovementioned treatments. It is explicitly shown that TD-1 and TD-2 have unreasonable vacuum limits, and their pressure at the minimum of energy (free energy at finite temperature) per baryon deviates obviously from zero, contradicting standard thermodynamics. The existence of an additional term to the thermodynamic potential density in TD-4 depends on whether the quark mass scaling satisfies the Cauchy condition that ensures the integrability of the relevant path integral. Unfortunately, however, neither Eq. (1) nor Eq. (2) meets the requirement. Therefore, the added term in TD-4 for thermodynamic consistency does not exist for the presently known quark mass scaling, and consequently TD-4 itself violates the Maxwell integrability condition. On the other hand, TD-3 with effective chemical potential interpretation can naturally give consistent vacuum limits, and the pressure at the minimum energy (free energy at finite temperature) is exactly zero. At the same time, all expressions in TD-3 are given explicitly without any integral, and more importantly, the Maxwell condition are fulfilled.

Recently, there is much progress in the measurement of compact stars with mass about 2 times the solar mass $\left(2 M_{\odot}\right)$ [59, 60]. It is shown that the reciprocity scaling in Eq. (1) together with TD-2 generates more massive strange stars than in the bag model [58]. However, the thermodynamic treatment, TD-2 used there, suffers from thermodynamic inconsistency, as the authors also noticed. On the other hand, the thermodynamically consistent TD-3 with the cubic-root scaling can describe stars with radii even smaller than in the bag model [16], but the maximum mass was normally much smaller than $2 M_{\odot}$ [46]. This case is mainly because the presently known quark mass scaling, either the reciprocity scaling or the cubic-root scaling, merely takes account of the confinement interaction, while the important perturbative interactions are unable to be included.

It is, therefore, our second purpose in the present paper to look for a new quark mass scaling which considers both the confinement and leading-order perturbative interactions. We show that this new scaling with TD-3 can describe quark stars with both low and high maximum masses, depending on the confinement and perturbative strength parameters. If SQM is absolutely stable, the maximum mass is as large as 2 times the solar mass, consistent with the recent measurements [59, 60].

In Sec. III, we first compare the four thermodynamic treatments. Then in Sec. III we present a fully consistent derivation of the thermodynamic treatment that will be used in the present paper. After arriving at a new quark mass scaling which includes both confinement and leading-order perturbative interactions in Sec. IV. the properties of SQM and the mass radius relation of strange stars are calculated in the new scaling in Secs. $\nabla$ and [VI] A summary is given in the final Sec. VII]

\section{INCONSISTENCY OF THE RECENT THERMODYNAMIC TREATMENT}

For comparison purpose, let us start from the fundamental differentiation equation of standard thermodynamics,

$$
\mathrm{d} \bar{E}=T \mathrm{~d} \bar{S}-P \mathrm{~d} V+\sum_{i} \mu_{i} \mathrm{~d} \bar{N}_{i}
$$

where $\bar{E}$ is the internal energy, $T$ the temperature, $\bar{S}$ the entropy, $\bar{N}_{i}$ the particle number of particle type $i$, 
and $\mu_{i}$ the corresponding chemical potential. The three terms on the right are, respectively, the three ways of increasing the system internal energy, i.e., heat transfer, doing work, and particle exchange.

Defining the free energy

$$
\bar{F} \equiv \bar{E}-T \bar{S},
$$

Eq. (3) then becomes

$$
\mathrm{d} \bar{F}=-\bar{S} \mathrm{~d} T-P \mathrm{~d} V+\sum_{i} \mu_{i} \mathrm{~d} \bar{N}_{i} .
$$

Similarly using the thermodynamic potential,

$$
\bar{\Omega} \equiv \bar{F}-\sum_{i} \mu_{i} \bar{N}_{i}=\bar{E}-T \bar{S}-\sum_{i} \mu_{i} \bar{N}_{i},
$$

then Eq. (3) or Eq. (5) becomes

$$
\mathrm{d} \bar{\Omega}=-\bar{S} \mathrm{~d} T-P \mathrm{~d} V-\sum_{i} \bar{N}_{i} \mathrm{~d} \mu_{i}
$$

In Ref. [48], the quark mass was assumed to be an intrinsic degree of freedom, and Eq. (7) was modified to

$$
\mathrm{d} \bar{\Omega}=-\bar{S} \mathrm{~d} T-P \mathrm{~d} V-\bar{N} \mathrm{~d} \mu+X \mathrm{~d} m^{*},
$$

when the system has only one type of particles and the particle mass is density and/or temperature dependent, i.e.,

$$
m^{*}=m^{*}\left(T, n_{\mathrm{b}}\right) .
$$

Equations (7) and (8) have significantly different thermodynamic consequences. In the latter case, all the thermodynamic formulas have the same form as these of a free system, as in Eq. (45) of Ref. [48], i.e.,

$$
\begin{array}{ll}
\bar{S}=-\left(\frac{\partial \bar{\Omega}}{\partial T}\right)_{V, \mu, m}, & P=-\left(\frac{\partial \bar{\Omega}}{\partial V}\right)_{T, \mu, m}, \\
\bar{N}=-\left(\frac{\partial \bar{\Omega}}{\partial \mu}\right)_{T, V, m}, & X=\left(\frac{\partial \bar{\Omega}}{\partial m}\right)_{T, V, \mu} .
\end{array}
$$

In physics, the effective mass is a phenomenological concept. It is usually introduced to meet some special purposes. For example, in the relativistic mean-field theory, an effective nucleon mass, $M^{*}=M_{\mathrm{N}}-g \phi$ with $g$ the coupling and $\phi$ the scalar field, was introduced to make the equation of motion become the Dirac equation [61]; in the NJL model, the quark's effective mass was introduced as $m_{q}^{*}=M_{q}-G\langle\bar{q} q\rangle$ [62]; to avoid complexity of nonlocality in the many-body theory of the early stage, the interacting nucleon was treated as a particle with kinetic energy $E_{k}=p^{2} /\left(2 M^{*}\right)+a$ where the effective mass satisfies $1 / M^{*}=1 / M+2 b[\underline{63}$; in quantum hadrondynamics, an effective mass of the nucleon was determined by a self-consistent equation [64]; etc.

In the present case, the density and/or temperature dependent quark mass is also conceptually effective. It is logically contradictory to assume the quark mass intrinsic while it is, in fact, dependent on, or determined

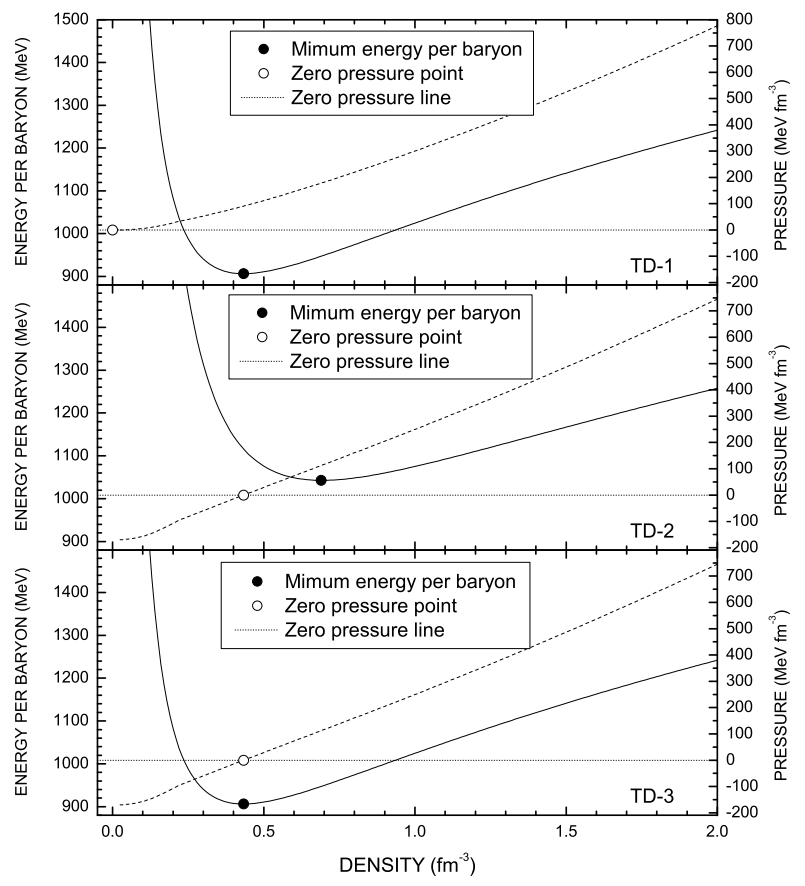

FIG. 1. Comparison of the thermodynamic treatments TD-1 (upper panel), TD-2 (middle panel), and TD-3 (lowest panel). The quark mass scaling parameters are the same as in Ref. [48] for Figs. 1 and 2 there. The energy per baryon and pressure

\begin{tabular}{|c|c|c|c|c|c|}
\hline & $\begin{array}{l}\mathcal{E}_{\min } \\
(\mathrm{MeV})\end{array}$ & $\begin{array}{l}\text { Density } \\
\left(\mathrm{fm}^{-3}\right)\end{array}$ & $\begin{array}{c}\text { Pressure } \\
\left(\mathrm{MeV} \mathrm{fm}^{-3}\right)\end{array}$ & $\begin{array}{c}n_{0} \\
\left(\mathrm{fm}^{-3}\right)\end{array}$ & $\begin{array}{c}\mathcal{E}_{0} \\
(\mathrm{MeV})\end{array}$ \\
\hline TD-1 & 906.3 & 0.433 & 90.7 & 0 & $\infty$ \\
\hline TD-2 & 1041.8 & 0.692 & 113.2 & 0.433 & 1116.2 \\
\hline TD-3 & 906.3 & 0.433 & 0 & 0.433 & 906.3 \\
\hline
\end{tabular}
are, respectively, on the left and right axis. The pressure is obviously nonzero at the minimum energy per baryon for TD-1 and TD-2, and exactly zero for TD-3.

TABLE I. Pressure at the minimum of energy per baryon calculated by the different thermodynamic treatment approaches TD-1 44, 48], TD-2 45], and TD-3.

by, the medium state variables (the density and/or temperature). The thermodynamic treatment derived from Eq. (8) is thus inevitably inconsistent with the standard thermodynamics.

To explicitly show the inconsistency, we have proved a necessary condition in the Appendix. At zero temperature, the condition is

$$
\Delta=P-n_{\mathrm{b}}^{2} \frac{\mathrm{d}}{\mathrm{d} n_{\mathrm{b}}}\left(\frac{E}{n_{\mathrm{b}}}\right)=0,
$$

where $P$ and $E$ are the model-given thermodynamic expressions. Any consistent thermodynamic treatment must ensure that $\Delta$ be zero at arbitrary density. Specifically, the pressure at the minimum energy per baryon must be zero.

In the upper panel of Fig. 1, we reproduce the Fig. 1 of Ref. [48], with the pressure added on the right axis. 


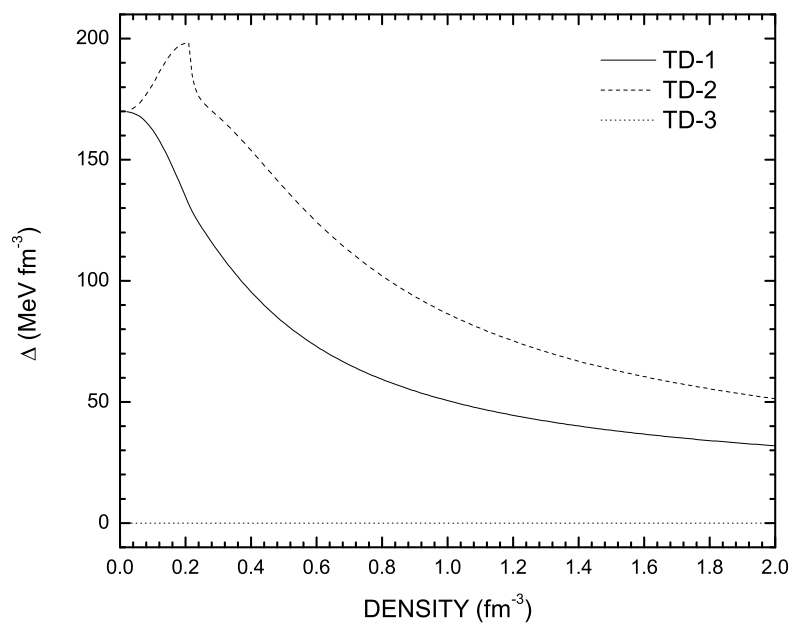

FIG. 2. The $\Delta$ as a function of density at zero temperature. The solid, dashed, and dotted lines are, respectively for the thermodynamic treatments TD-1, TD-2, and TD-3.

The cases for the second and third treatments are also given, respectively, in the middle and lowest panels for comparison. The minimum energy per baryon $\left(\mathcal{E}_{\min }\right)$, its position density, the pressure at the minimum, the position of zero pressure $\left(n_{0}\right)$, and the energy per baryon at the zero pressure $\left(\mathcal{E}_{0}\right)$ are listed in Table 1 . It is obviously seen that the pressure at the energy minimum is nonzero for TD-1 and TD-2, and they are exactly located at the same density for TD-3.

In Fig. 2, we give the $\Delta$ as a function of density with the same mass scaling and parameters [Eq. (1) with $m_{u 0}=m_{d 0}=0, m_{s 0}=150 \mathrm{MeV}, B^{1 / 4}=$ $170 \mathrm{MeV} \mathrm{fm}^{-3}$. Again, we see that both TD-1 and TD-2 cannot give zero $\Delta$, while TD-3 naturally gives $\Delta=0$ at the whole density region.

Aside from the nonzero $\Delta$ at arbitrary density and the nonzero pressure at the energy minimum, the vacua in TD-1 and TD-2 are also contradictory themselves. Using the reciprocity scaling in Eq. (1), one can easily obtain

$$
\begin{aligned}
& \lim _{n_{\mathrm{b}} \rightarrow 0} E_{1}=B, \lim _{n_{\mathrm{b}} \rightarrow 0} P_{1}=0 . \\
& \lim _{n_{\mathrm{b}} \rightarrow 0} E_{2}=2 B, \lim _{n_{\mathrm{b}} \rightarrow 0} P_{2}=-B . \\
& \lim _{n_{\mathrm{b}} \rightarrow 0} E_{3}=B,
\end{aligned}
$$

where $E_{i}$ and $P_{i}(i=1,2,3)$ are the corresponding energy density and pressure in the three thermodynamic treatments. The vacua in TD- 1 and TD- 2 violate the universal energy conservation law.

Therefore, modifying the fundamental thermodynamic equation causes unavoidable inconsistencies. In fact, one should not modify the fundamental equation (7). Instead, we should modify phenomenological models to meet its requirements. In the next section, we will explain such a scheme to make the previous thermodynamic treatment in full consistency with the standard thermodynamics.
Because the pressure in TD-1 cannot be negative, another treatment was recommended in Sec. V of Ref. 48]. The recommended treatment follows that in Ref. [49], where the total thermodynamic density was written as

$$
\Omega=\Omega_{0}\left(T,\left\{\mu_{i}\right\},\left\{m_{i}\right\}\right)+\Omega_{\mathrm{a}}\left(n_{\mathrm{b}}\right) .
$$

The functional form of $\Omega_{0}$ is the same as a free-particle system.

Adding a new term to the thermodynamic potential density is a good idea because the thermodynamic potential density changes when interactions set in. In the quasiparticle model, the added term can be selfconsistently obtained when the particle mass depends solely on temperature [35], or on respective chemical potentials $\left[m_{i}^{*}=m_{i}^{*}\left(\mu_{i}\right)\right.$ when the chemical potentials are not coupled] [36], or even the finite size effects are considered 65 .

In the case of density and/or temperature dependent masses, however, chemical potentials are surely coupled. We therefore need to check if the added term really exists for the presently known quark mass scaling.

Let's first see how the added term is determined in the literature [48, 49]. Similarly as in the quasiparticle model, the extra term was chosen to be determined by

$$
\frac{\mathrm{d} \Omega_{\mathrm{a}}}{\mathrm{d} n_{\mathrm{b}}}=-\sum_{i} \frac{\partial \Omega_{0}}{\partial m_{i}} \frac{\mathrm{d} m_{i}}{\mathrm{~d} n_{\mathrm{b}}}
$$

so that the particle number density was still given by

$$
n_{i}=-\frac{\partial \Omega_{0}}{\partial \mu_{i}}=\frac{g_{i}}{6 \pi^{2}}\left(\mu_{i}^{2}-m_{i}^{2}\right)^{3 / 2} .
$$

Then from Eq. (15) the added term was obtained as

$$
\Omega_{\mathrm{a}}\left(n_{\mathrm{b}}\right)=-\int_{\rho_{c}}^{n_{\mathrm{b}}} \sum_{i} \frac{\partial \Omega_{0}}{\partial m_{i}} \frac{\mathrm{d} m_{i}}{\mathrm{~d} n_{\mathrm{b}}} \mathrm{d} n_{\mathrm{b}}+\Omega_{a}\left(\rho_{c}\right),
$$

where $\rho_{c}$ is an integral constant.

In the integrand of Eq. (17), there is not only the argument $n_{\mathrm{b}}$, but also the chemical potentials $\mu_{i}(i=u, d, s)$. Because these chemical potentials are not constants [otherwise one can inverse Eq. (17) to go back to the bag model], they must be determined as functions of the density:

$$
\mu_{i}=\mu_{i}\left(n_{\mathrm{b}}\right) .
$$

In Refs. [48, 49], the functions $\mu_{i}\left(n_{\mathrm{b}}\right)$ were chosen by solving Eqs. (69)-(71). Because the thermodynamic potential density is a state quantity, however, the integration should be independent of the special path. In the following, we show that only when the Cauchy condition is satisfied, can one obtain the additional term by choosing a special path.

In fact, to eliminate the extra term in the expression of particle numbers due to the density dependence of quark masses, one must require

$$
\frac{\partial \Omega_{a}}{\partial \mu_{i}}=-\sum_{k} \frac{\partial \Omega_{0}}{\partial m_{k}} \frac{\mathrm{d} m_{k}}{\mathrm{~d} n_{\mathrm{b}}} \frac{\partial n_{\mathrm{b}}}{\partial \mu_{i}} .
$$


Namely, the additional term should be given by a path integral as

$$
\begin{aligned}
\Omega_{\mathrm{a}} & =-\int_{\mu_{0}}^{\mu} \sum_{i} \frac{\partial \Omega_{a}}{\partial \mu_{i}} \mathrm{~d} \mu_{i} \\
& =-\int_{\mu_{0}}^{\mu}\left(\sum_{k} \frac{\partial \Omega_{0}}{\partial m_{k}} \frac{\mathrm{d} m_{k}}{\mathrm{~d} n_{\mathrm{b}}}\right) \sum_{i} \frac{\partial n_{\mathrm{b}}}{\partial \mu_{i}} \mathrm{~d} \mu_{i} .
\end{aligned}
$$

When one chooses a special path such as that in Eq. (18), Eq. (20) leads to Eq. (17). Because $\Omega_{\mathrm{a}}$ is a state function of the independent state quantities $\mu_{i}$ $(i=u, d$, and $s)$, the path integral on the right-hand side of Eq. (20) should be path independent. For this the famous Cauchy theorem must be satisfied:

$$
\frac{\partial^{2} \Omega_{\mathrm{a}}}{\partial \mu_{j} \partial \mu_{i}}=\frac{\partial^{2} \Omega_{\mathrm{a}}}{\partial \mu_{i} \partial \mu_{j}}
$$

Substituting the right-hand side of Eq. (19) then gives

$$
\frac{\sum_{k} \frac{\partial^{2} \Omega_{0}}{\partial \mu_{i} \partial m_{k}} \frac{\mathrm{d} m_{k}}{\mathrm{~d} n_{\mathrm{b}}}}{\partial n_{\mathrm{b}} / \partial \mu_{i}}=\frac{\sum_{k} \frac{\partial^{2} \Omega_{0}}{\partial \mu_{j} \partial m_{k}} \frac{\mathrm{d} m_{k}}{\mathrm{~d} n_{\mathrm{b}}}}{\partial n_{\mathrm{b}} / \partial \mu_{j}}
$$

On application of Eq. (16), one has

$$
\frac{\partial n_{\mathrm{b}}}{\partial \mu_{i}}=\frac{\mu_{i} \sqrt{\mu_{i}^{2}-m_{i}^{2}}}{\frac{6 \pi^{2}}{g}+\sum_{k} m_{k} \sqrt{\mu_{k}^{2}-m_{k}^{2}} \frac{\mathrm{d} m_{k}}{\mathrm{~d} n_{\mathrm{b}}}} .
$$

Then one can obtain

$$
\frac{m_{u}}{\mu_{u}} \frac{\mathrm{d} m_{u}}{\mathrm{~d} n_{\mathrm{b}}}=\frac{m_{d}}{\mu_{d}} \frac{\mathrm{d} m_{d}}{\mathrm{~d} n_{\mathrm{b}}}=\frac{m_{s}}{\mu_{s}} \frac{\mathrm{d} m_{s}}{\mathrm{~d} n_{\mathrm{b}}} .
$$

Unfortunately, however, neither the reciprocity scaling [42] nor the cubic-root scaling satisfies the Cauchy condition (24). An even more general form of $m_{i}=$ $m_{i 0}+m_{\mathrm{I}}\left(n_{\mathrm{b}}\right)$ cannot do so. Therefore, the additional term $\Omega_{\mathrm{a}}$ does not exist for the presently known density and/or temperature dependent quark mass scaling. We should therefore look for another more convenient treatment that is in agreement with the fundamental Eq. (77), or equivalently, Eq. (5).

\section{SELF-CONSISTENT THERMODYNAMICS WITH DENSITY AND/OR TEMPERATURE DEPENDENT PARTICLE MASSES}

Because the system we are studying has density and/or temperature dependent masses, it is naturally convenient to choose the temperature $T$, the densities $n_{i}$, and the volume $V$ as the independent state variables. Therefore, we rewrite the fundamental Eq. (5) as

$$
\mathrm{d} F=-S \mathrm{~d} T+\left(-P-F+\sum_{i} \mu_{i} n_{i}\right) \frac{\mathrm{d} V}{V}+\sum_{i} \mu_{i} \mathrm{~d} n_{i}
$$

where $F \equiv \bar{F} / V, S \equiv \bar{S} / V, n_{i} \equiv \bar{N}_{i} / V$ are, respectively, the free-energy density, the entropy density, and the particle number densities.

For an infinitely large system such as quark matter, the free-energy density has nothing to do with the volume. In this case, we have

$$
P=-F+\sum_{i} \mu_{i} n_{i}
$$

and

$$
\mathrm{d} F=-S \mathrm{~d} T+\sum_{i} \mu_{i} \mathrm{~d} n_{i} .
$$

Now we try to establish a thermodynamic treatment in full agreement with this fundamental equation. Because both TD-1 and TD-2 do not give zero pressure at the energy minimum, they should naturally be discarded. The model we are trying to build resembles, in many aspects, the third treatment TD-3.

At zero temperature, the energy density of a free quark system is

$$
E_{0}=\sum_{i} g_{i} \int_{0}^{\nu_{i}} \sqrt{p^{2}+m_{i}^{2}} \frac{p^{2} \mathrm{~d} p}{2 \pi^{2}}
$$

where $\nu_{i}$ is the particle type $i$ 's Fermi momentum which is connected to the corresponding particle number density by

$$
n_{i}=g_{i} \int_{0}^{\nu_{i}} \frac{p^{2} \mathrm{~d} p}{2 \pi^{2}}=\frac{g_{i} \nu_{i}^{3}}{6 \pi^{2}} .
$$

When the quarks interact with each other, we want to include the interaction effect with a density dependent mass as

$$
m_{i}=m_{i 0}+m_{\mathrm{I}},
$$

where $m_{\mathrm{I}}$ is a density dependent quantity. We demand that the system energy density still has the same form as in Eq. (28), i.e., $E=E_{0}$, which is also the original idea in Ref. [42]. This is possible because $E_{0}$ is an increasing function of the particle masses. To distinguish with other mass concepts, we call such a mass an equivalent mass [66]. With the equivalent mass, both the energy density and particle number densities have the same form as a free particle system while only the particle number densities keep unchanged in the quasiparticle model. We call such a model an equiparticle model. The corresponding pressure in the equiparticle model can then be easily deduced according to the fundamental thermodynamics, as in Sec. II of Ref. [2]. Here we do not repeat the derivation. The key point is that the Fermi momentum $\nu_{i}$ is not directly linked to the chemical potential $\mu_{i}$ by $\nu_{i}=\sqrt{\mu_{i}^{2}-m_{i}^{2}}$; instead, it is connected to an effective chemical potential $\mu_{i}^{*}$ by $\nu_{i}=\sqrt{\mu_{i}^{* 2}-m_{i}^{2}}$, while the relation between the effective and real chemical potentials are determined by the fundamental differential 
equation (27), and, consequently, the pressure, as well as the thermodynamic potential density have an additional term due to the density dependence of quark masses.

At finite temperature, the concept of the Fermi momentum is not as useful as in the case of zero temperature. We should directly use the concept of effective chemical potentials. Also the characteristic function should be changed from the energy to the free energy. Therefore, we write the free-energy density of the system the same as that of a free system with equivalent particle mass $m_{i}\left(T, n_{\mathrm{b}}\right)$ and effective chemical potentials $\mu_{i}^{*}$ at temperature $T$, i.e.,

$$
F=\Omega_{0}\left(T,\left\{\mu_{i}^{*}\right\},\left\{m_{i}\right\}\right)+\sum_{i} \mu_{i}^{*} n_{i} .
$$

Please note the arguments in $\Omega_{0}$ : the position of free particle's chemical potentials have been replaced with the effective chemical potentials $\mu_{i}^{*}$, i.e.,

$$
\Omega_{0}=\Omega_{0}\left(T,\left\{\mu_{i}^{*}\right\},\left\{m_{i}\right\}\right)
$$

is the thermodynamic potential density of a free system with the particle masses $m_{i}\left(T, n_{\mathrm{b}}\right)$ and chemical potentials $\mu_{i}^{*}$. Because the independent state variables are $\left(T, V,\left\{n_{i}\right\}\right)$, not including $\mu_{i}^{*}$, we should also choose how to connect $\mu_{i}^{*}$ to the independent variables. Here we choose to connect the effective chemical potentials to particle number densities by

$$
n_{i}=-\frac{\partial}{\partial \mu_{i}^{*}} \Omega_{0}\left(T,\left\{\mu_{i}^{*}\right\},\left\{m_{i}\right\}\right),
$$

which are also the choice of many previous thermodynamic treatments, but the real chemical potentials have been replaced with effective ones here to ensure the thermodynamic consistency.

To derive other thermodynamic quantities, let us differentiate Eq. (31) to give

$$
\mathrm{d} F=\mathrm{d} \Omega_{0}+\sum_{i} n_{i} \mathrm{~d} \mu_{i}^{*}+\sum_{i} \mu_{i}^{*} \mathrm{~d} n_{i}
$$

where

$$
\mathrm{d} \Omega_{0}=\frac{\partial \Omega_{0}}{\partial T} \mathrm{~d} T+\sum_{i} \frac{\partial \Omega_{0}}{\partial \mu_{i}^{*}} \mathrm{~d} \mu_{i}^{*}+\sum_{i} \frac{\partial \Omega_{0}}{\partial m_{i}} \mathrm{~d} m_{i}
$$

with

$$
\mathrm{d} m_{i}=\frac{\partial m_{i}}{\partial T} \mathrm{~d} T+\sum_{j} \frac{\partial m_{i}}{\partial n_{j}} \mathrm{~d} n_{j} .
$$

On application of Eqs. (33), (35), and (36), Eq. (34) becomes

$$
\begin{aligned}
\mathrm{d} F= & \left(\frac{\partial \Omega_{0}}{\partial T}+\sum_{i} \frac{\partial \Omega_{0}}{\partial m_{i}} \frac{\partial m_{i}}{\partial T}\right) \mathrm{d} T \\
& +\sum_{i}\left(\mu_{i}^{*}+\sum_{j} \frac{\partial \Omega_{0}}{\partial m_{j}} \frac{\partial m_{j}}{\partial n_{i}}\right) \mathrm{d} n_{i} .
\end{aligned}
$$

Comparing this equation with Eq. (27), we immediately have the entropy density

$$
S=-\frac{\partial \Omega_{0}}{\partial T}-\sum_{i} \frac{\partial \Omega_{0}}{\partial m_{i}} \frac{\partial m_{i}}{\partial T}
$$

and the true chemical potential

$$
\mu_{i}=\mu_{i}^{*}+\sum_{j} \frac{\partial \Omega_{0}}{\partial m_{j}} \frac{\partial m_{j}}{\partial n_{i}} \equiv \mu_{i}^{*}-\mu_{\mathrm{I}} .
$$

The pressure can be obtained by substituting Eq. (31) into Eq. (26), giving $P=-\Omega_{0}+\sum_{i}\left(\mu_{i}-\mu_{i}^{*}\right) n_{i}$, i.e.,

$$
P=-\Omega_{0}+\sum_{i, j} \frac{\partial \Omega_{0}}{\partial m_{j}} n_{i} \frac{\partial m_{j}}{\partial n_{i}} .
$$

The energy density is obtained by substituting Eqs. (31) and (38) into $E=F+T S$ as

$$
E=\Omega_{0}-\sum_{i} \mu_{i}^{*} \frac{\partial \Omega_{0}}{\partial \mu_{i}^{*}}-T \frac{\partial \Omega_{0}}{\partial T}-T \sum_{i} \frac{\partial \Omega_{0}}{\partial m_{i}} \frac{\partial m_{i}}{\partial T},
$$

while the real thermodynamic potential density is

$$
\Omega=F-\sum_{i} \mu_{i} n_{i}=\Omega_{0}-\sum_{i, j} \frac{\partial \Omega_{0}}{\partial m_{j}} n_{i} \frac{\partial m_{j}}{\partial n_{i}} .
$$

For a given set of the independent state variables $T$ and $n_{i}$, the effective chemical potential $\mu_{i}^{*}$ is obtained by solving the equation(s) in Eq. (33). Then other thermodynamic quantities can be calculated, respectively, by Eqs. (38)-(42) if the temperature and density dependence of the quark masses is known.

From Eqs. (40) and (42), one finds that the normal relation $P=-\Omega$ still holds. In fact, all the basic relations of standard thermodynamics are maintained in the present treatment. The $\Omega_{0}$, as seen from the derivation process, serves merely as an intermediate quantity, while other thermodynamic quantities are derived and expressed in its functional form. In the following, for example, we list formulas for the two specially important cases at zero temperature.

\section{A. Color-flavor locking with density-dependent particle masses}

The color-flavor locked phase is believed to exist at extremely high density [67, 68]. In the MIT bag model, one has known how to construct the thermodynamic density long ago [69, 70, 80]. To consider the medium effect with density-dependent quark masses, one can similarly construct the thermodynamics density [71, 72].

The free particle contribution is

$$
\begin{aligned}
\Omega_{0}= & \sum_{q} \frac{3}{\pi^{2}} \int_{0}^{\nu}\left(\sqrt{p^{2}+m_{q}^{2}}-\mu_{q}^{*}\right) p^{2} \mathrm{~d} p \\
& +\frac{3 \Delta^{2}}{\pi^{2}} \bar{\mu}^{2}+B .
\end{aligned}
$$


where the chemical potentials have been replaced with the effective ones to consider medium effect with density dependent particle masses, the second term is the paring contribution with $\bar{\mu}=\left(\mu_{u}^{*}+\mu_{d}^{*}+\mu_{s}^{*}\right) / 3$, and the last term is added if one would also like to include the vacuum contribution. The common Fermi momentum in the first term is obtained by minimizing $\Omega_{0}$ at fixed $\mu_{q}^{*}$, i.e., $\partial \Omega_{0} / \partial \nu=0$ which gives

$$
\sum_{q} \sqrt{\nu^{2}+m_{q}^{2}}=3 \bar{\mu}
$$

All other thermodynamic quantities can now be directly obtained from the above formulas. They are the same as in Ref. 71], and including finite-size effects in Ref. [72], with the emphasis that the chemical potentials there be regarded as effective ones according to the above consistent thermodynamic derivations.

\section{B. The unpaired case at zero temperature}

For the unpaired SQM at finite temperature, we have the same formulas as in Ref. [2], or including finite-size effects in Ref. 47]. At zero temperature, the formulas are still the same as those in Ref. [46]. Again, the chemical potentials there should be regarded as effective ones.

For the convenience of getting a new quark mass scaling in the next section, we give the free unpaired particle contribution:

$$
\Omega_{0}=-\sum_{i} \frac{g_{i}}{24 \pi^{2}}\left[\mu_{i}^{*} \nu_{i}\left(\nu_{i}^{2}-\frac{3}{2} m_{i}^{2}\right)+\frac{3}{2} m_{i}^{4} \ln \frac{\mu_{i}^{*}+\nu_{i}}{m_{i}}\right] .
$$

Correspondingly, we have the particle number density

$$
n_{i}=\frac{g_{i}}{6 \pi^{2}}\left(\mu_{i}^{* 2}-m_{i}^{2}\right)^{3 / 2}=\frac{g_{i} \nu_{i}^{3}}{6 \pi^{2}}
$$

the relation between the real and effective chemical potentials

$$
\mu_{i}=\mu_{i}^{*}+\frac{1}{3} \frac{\partial m_{\mathrm{I}}}{\partial n_{\mathrm{b}}} \frac{\partial \Omega_{0}}{\partial m_{\mathrm{I}}} \equiv \mu_{i}^{*}-\mu_{\mathrm{I}}
$$

and the pressure

$$
P=-\Omega_{0}+n_{\mathrm{b}} \frac{\partial m_{\mathrm{I}}}{\partial n_{\mathrm{b}}} \frac{\partial \Omega_{0}}{\partial m_{\mathrm{I}}}=-\Omega_{0}-3 n_{\mathrm{b}} \mu_{\mathrm{I}} .
$$

In Eqs. (45)-(48),

$$
\nu_{i}=\sqrt{\mu_{i}^{* 2}-m_{i}^{2}}
$$

is the Fermi momentum of particle type $i$, while the derivative of $\Omega_{0}$ with respect to the interacting quark mass is

$$
\frac{\partial \Omega_{0}}{\partial m_{\mathrm{I}}}=\sum_{i} \frac{g_{i} m_{i}}{4 \pi^{2}}\left[\mu_{i}^{*} \nu_{i}-m_{i}^{2} \ln \frac{\mu_{i}^{*}+\nu_{i}}{m_{i}}\right] .
$$

For future convenience, we define a holistic Fermi momentum as

$$
\nu \equiv\left(\frac{1}{N_{\mathrm{f}}} \sum_{q} \nu_{q}^{3}\right)^{1 / 3},
$$

where the summation index $q$ goes over all quark flavors involved, and $N_{\mathrm{f}}$ is the quark flavor number. Equation (51) means $\nu$ is the subtriplicate of the averaged cubic Fermi momentum. With a view to Eq. (46) and the definition of the baryon number density $n_{\mathrm{b}}=\sum_{q} n_{q} / 3$, Eq. (51) can naturally be linked to density by

$$
\nu=\left(\frac{3 \pi^{2}}{N_{\mathrm{f}}} n_{\mathrm{b}}\right)^{1 / 3}, \quad n_{\mathrm{b}}=\frac{N_{\mathrm{f}}}{3 \pi^{2}} \nu^{3} .
$$

Obviously, $\nu$ has the dimension of energy. It can therefore be used as an energy scale of cold quark matter. For the color-flavor locked case, it equals to the common fermi momentum.

\section{QUARK MASS SCALING WITH LINEAR CONFINEMENT AND LEADING-ORDER PERTURBATIVE INTERACTIONS}

In order to get an appropriate equivalent mass, we carry out the similar procedure as was done in Ref 73], namely we expand the equivalent mass to a Laurant series of the holistic Fermi momentum $\nu$, and take the leading term in both directions:

$$
m_{\mathrm{I}}=\frac{a_{-1}}{\nu}+a_{1} \nu
$$

We will soon see that the first term corresponds to the linear confinement, while the second term is responsible for the leading-order perturbative interactions.

At lower density, the first term becomes infinitely large when the holistic Fermi momentum, or the density, approaches to zero. Therefore, Eq. (53) becomes $m_{\mathrm{I}}=a_{-1} / \nu$ at lower density. On the other hand, we have already known that the lower density behavior is $m_{\mathrm{I}}=D / n_{\mathrm{b}}^{1 / 3}$ with the confinement parameter $D$ connected to the string tension $\sigma_{0}$, the chiral restoration density $\rho^{*}$, and the sum of the vacuum chiral condensates $\sum_{q}\langle\bar{q} q\rangle_{0}$ by

$$
D \sim \frac{3(2 / \pi)^{1 / 3} \sigma_{0} \rho^{*}}{-\sum_{q}\langle\bar{q} q\rangle_{0}}
$$

Although we cannot use this formula to exactly calculate the $D$ value due to the uncertainties in relevant quantities, we do know that $D$ is a low energy parameter, and that $\sqrt{D}$ approximately is in the range of $(147,270) \mathrm{MeV}$ [47]. Equating $a_{-1} / \nu$ and $D / n_{\mathrm{b}}^{1 / 3}$, we immediately find

$$
a_{-1}=D\left(\frac{3 \pi^{2}}{N_{\mathrm{f}}}\right)^{1 / 3}
$$


At higher density, the second term in Eq. (53) dominates. A little later, we show that the coefficient $a_{1}$ runs with the strong coupling constant $\alpha \equiv \alpha_{s} / \pi=g^{2} / 4 \pi^{2}$ according to the equation

$$
\sqrt{1+a_{1}^{2}}\left(1+\frac{a_{1}^{2}}{2}\right)-\frac{a_{1}^{4}}{2} \ln \frac{1+\sqrt{1+a_{1}^{2}}}{a_{1}}=(1-2 \alpha)^{-1 / 3} .
$$

In fact, we can prove Eq. (56) by comparing the present model at higher density with the perturbation results.

There are several expressions for the pressure of a cold quark plasma, e.g., those from the hard-thermal-loop perturbation theory [74] and from the weak-coupling expansion $[75$ 77]. Although they are different in higher orders, the leading term is identical, i.e.,

$$
\begin{aligned}
P & =\frac{N_{\mathrm{f}} \mu^{4}}{4 \pi^{2}}(1-2 \alpha), \\
n_{\mathrm{b}} & =\frac{N_{\mathrm{f}} \mu^{3}}{3 \pi^{2}}(1-2 \alpha) .
\end{aligned}
$$

At high density, because of the weak chemical equilibrium condition $\mu_{u}+\mu_{e}=\mu_{d}=\mu_{s}$ and the quark current masses being unimportant, we consider the flavorsymmetric case in the present model when $m_{u 0}=m_{d 0}=$ $m_{s 0}=0, \mu_{u}=\mu_{d}=\mu_{s} \equiv \mu, \nu_{u}=\nu_{d}=\nu_{s}=\nu$, and $m_{i}=m_{\mathrm{I}}$. At high density, the second term on the right-hand side of Eq. (53) gives $m_{\mathrm{I}}=a_{1} \nu$. Accordingly, we have $\mu^{*}=\sqrt{\nu^{2}+m_{\mathrm{I}}^{2}}=\sqrt{1+a_{1}^{2}} \nu$, and $\mathrm{d} m_{\mathrm{I}} / \mathrm{d} \nu=a_{1}+\nu \mathrm{d} a_{1} / \mathrm{d} \nu \approx a_{1}$.(The second term is of higher order in the coupling, and can thus be ignored.) On application of these facts, Eq. (47) gives the relation between the actual and effective chemical potentials as

$$
\mu^{*}=\mu\left(1+\frac{a_{1}^{2}}{2}-\frac{a_{1}^{4}}{2 \sqrt{1+a_{1}^{2}}} \ln \frac{1+\sqrt{1+a_{1}^{2}}}{a_{1}}\right)^{-1}
$$

and the Fermi momentum is then

$$
\nu=\mu\left[\sqrt{1+a_{1}^{2}}\left(1+\frac{a_{1}^{2}}{2}\right)-\frac{a_{1}^{4}}{2} \ln \frac{1+\sqrt{1+a_{1}^{2}}}{a_{1}}\right]^{-1} .
$$

Substituting Eq. (60) into the second equality of Eq. (52) and then comparing with Eq. (58), or, equivalently, comparing Eq. (48) with Eq. (57) after using Eqs. (59) and (60) etc, we immediately obtain Eq. (56).

Equation (56) determines $a_{1}$ as a function of the strong coupling $\alpha$. Although the numerical calculation of the function is easy, an explicit expression of the functional form may be helpful. For this we can ignore the logarithmic term that is of the fourth order in $a_{1}$, giving

$$
a_{1}=\sqrt{\left(\frac{\sqrt[3]{a+\sqrt{a^{2}+27}}}{3}-\frac{1}{\sqrt[3]{a+\sqrt{a^{2}+27}}}\right)^{2}-1}
$$

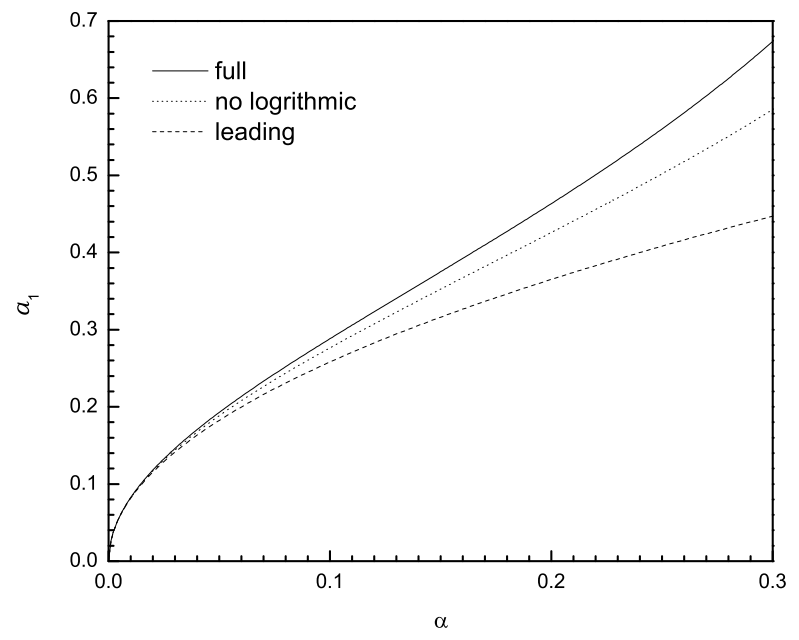

FIG. 3. Variation of the perturbation parameter $a_{1}$ with the strong coupling $\alpha=\alpha_{\mathrm{s}} / \pi$. The solid curve is solved from Eq. (56), the dotted curve is calculated by Eq. (61) which ignores the logarithmic term in Eq. (56), and the dashed line is the leading contribution given by Eq. 62).

with $a=27(1-2 \alpha)^{-1 / 3}$. Or, for simplicity, we just keep the leading term as

$$
a_{1}=\sqrt{\frac{2}{3} \alpha}
$$

The functions $a_{1}(\alpha)$ from Eqs. (56), (61), and (62) are given, respectively, by solid, dotted, and dashed curves in Fig. 3.

Now let us rewrite the quark equivalent mass as

$$
m_{i}=m_{i 0}+\frac{D}{n_{\mathrm{b}}^{1 / 3}}+C_{1} a_{1} n_{\mathrm{b}}^{1 / 3}
$$

which is obtained by substituting Eqs. (52) and (55) into Eq. (53). The factor $\left(3 \pi^{2} / N_{\mathrm{f}}\right)^{1 / 3} \equiv C_{1 \max }$ in the term proportional to the cubic-root density has been replaced with a parameter $C_{1}$. This is because we have ignored the quark current masses in the derivation of the mass scaling. If the finite current quark masses were included, one would find that the factor is smaller. Also, other approaches might give different $C_{1}$ values, for example, when one considers the one-gluon-exchange interaction [56], or the isospin interaction [57]. Therefore, we choose $C_{1}$ as a phenomenological model parameter in the range of $\left|C_{1}\right|<\pi^{2 / 3} \approx 2.145$.

We have already known that the $a_{1}$ in Eq. (63) depend on the running coupling $\alpha$, i.e., $a_{1}=a_{1}(\alpha)$. Now we have to discuss how the strong coupling is running.

The running coupling satisfies the renormalizationgroup equation:

$$
\frac{\mathrm{d} \alpha}{\mathrm{d} \ln u^{2}}=\beta(\alpha)=\sum_{i} \beta_{i} \alpha^{i+2},
$$




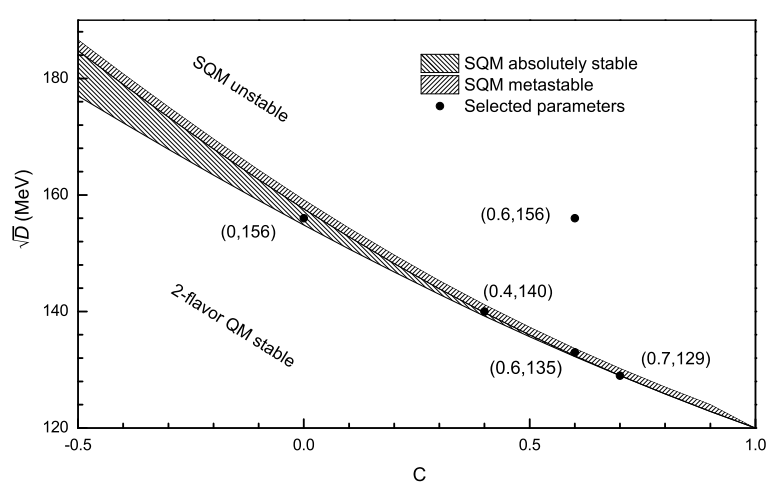

FIG. 4. Different range of the parameters $D$ and $C$. Within the shaded region, SQM is stable. In the upper-right region, SQM is unstable, while the lower-left is the forbidden region where two-flavor quark matter is stable.

where the beta functions, $\beta_{i}$, depend generally on which renormalization scheme is used. In the minimum subtraction scheme 78], they are known to the fourth order [79]. The first two beta functions, $\beta_{0}$ and $\beta_{1}$, are independent of renormalization schemes, i.e., $\beta_{0}=11 / 4-N_{\mathrm{f}} / 6$ and $\beta_{1}=51 / 8-19 N_{\mathrm{f}} / 24$.

Simply truncating the right-hand side of Eq. (64) to the first order in $\alpha$, one can easily find an inversely logarithmic solution. It is well known, however, this simple solution has an obviously too-large deviation from higherorder solutions. Recently, a fast convergent expression has been obtained by resummation over an infinite number of known terms into a compact form [80], and the leading contribution is

$$
\alpha=\frac{\beta_{0}}{\beta_{0}^{2} \ln \left(u^{2} / \Lambda^{2}\right)+\beta_{1} \ln \ln \left(u^{2} / \Lambda^{2}\right)},
$$

where $\Lambda$ is a QCD scale parameter, and we take $\Lambda=325$ $\mathrm{MeV}$ as determined in Ref. [80].

Another method is to use the analytic coupling constant in the one-loop approximation [81]

$$
\alpha=\frac{1}{\beta_{0}}\left[\frac{1}{\ln \left(u^{2} / \Lambda^{2}\right)}+\frac{1}{1-u^{2} / \Lambda^{2}}\right] .
$$

Both Eqs. (65) and (66) indicate that the coupling runs logarithmically. The running rate is thus much slower. Therefore, we can use an averaged constant coupling to rewrite Eq. (63) as

$$
m_{i}=m_{i 0}+\frac{D}{n_{\mathrm{b}}^{1 / 3}}+C n_{\mathrm{b}}^{1 / 3}
$$

where $C_{1} a_{1}$ has been grouped to be replaced with an averaged constant $C$.

From Eq. (66), it is easy to show that the maximum $\alpha$ value is $1 / \beta_{0}$. Thus the maximum value of $a_{1}$ is $\sqrt{2 /\left(3 \beta_{0}\right)}$ according to Eq. (62). Consequently, we have

$$
C<\left(\frac{3 \pi^{2}}{N_{\mathrm{f}}}\right)^{1 / 3} \sqrt{\frac{2}{3 \beta_{0}}} \approx 1.1676
$$

In Fig. 4, we show the different regions of the parameters $C$ and $D$ when taking $m_{u 0}=5 \mathrm{MeV}, m_{d 0}=10$ $\mathrm{MeV}$, and $m_{s 0}=100 \mathrm{MeV}$. The lower-left region is forbidden where two-flavor quark matter is stable, while in the up-right region SQM is unstable. Only in the shaded region, SQM is absolute or metastable. In the following calculations with Eq. (67), we will take the typical sets of $(C, \sqrt{D})$ pairs where $C$ is dimensionless and $\sqrt{D}$ is in $\mathrm{MeV}:(0.7,129),(0.6,135),(0.4,140),(0,156),(0.6,156)$. These parameter pairs are indicated in Fig. 4 with solid dots.

We would like to emphasize that the equivalent mass is in principle connected to the in-medium chiral condensates [66, 73, 82], and is thus different from various effective masses. In a NJL-type (or SchwingerDyson, relativistic-mean-field, ...) description of interacting quarks, one has contributions that affect the mass via scalar densities and the chemical potential through vector densities. In the present approach, an equivalent mass includes contributions from both the scalar and vector fields. It was explicitly shown in Ref. [66], in the context of symmetric nuclear matter and to leading order in quantum hadrondynamics, that the nucleon's effective mass involves only the scalar field $\sigma$, while its equivalent mass is linked to both the scalar $\sigma$ field and the Lorentz vector field $\omega$.

\section{PROPERTIES OF STRANGE QUARK MATTER}

As usually done, we assume SQM to be composed of up $(u)$, down $(d)$, and strange $(s)$ quarks with charge neutrality maintained by the inclusion of electrons $(e)$ [10]. Due to the weak interactions such as $d, s \leftrightarrow u+e+\bar{\nu}_{e}$, $s+u \leftrightarrow u+d$, etc, the chemical potentials $\mu_{i}(i=$ $u, d, s, e$ ) satisfy the weak equilibrium conditions (neutrinos enter and leave the system freely, and the corresponding chemical potential has been taken to be zero):

$$
\mu_{u}+\mu_{e}=\mu_{d}=\mu_{s} .
$$

The charge neutrality condition reads

$$
\frac{2}{3} n_{u}-\frac{1}{3} n_{d}-\frac{1}{3} n_{s}-n_{e}=0,
$$

with the baryon number conservation

$$
\frac{1}{3}\left(n_{u}+n_{d}+n_{s}\right)=n_{\mathrm{b}}
$$

In the present thermodynamic treatment, the particle number densities $n_{i}(i=u, d, s, e)$ in Eqs. (70) and (71) are connected, by Eq. (46), to the effective chemical potentials $\mu_{i}^{*}$ which is linked to the real chemical potentials $\mu_{i}$ by Eq. (47). Therefore, Eqs. (69)-(71) are four equations about the four chemical potentials $\mu_{i}$ which can be solved out at given density. 


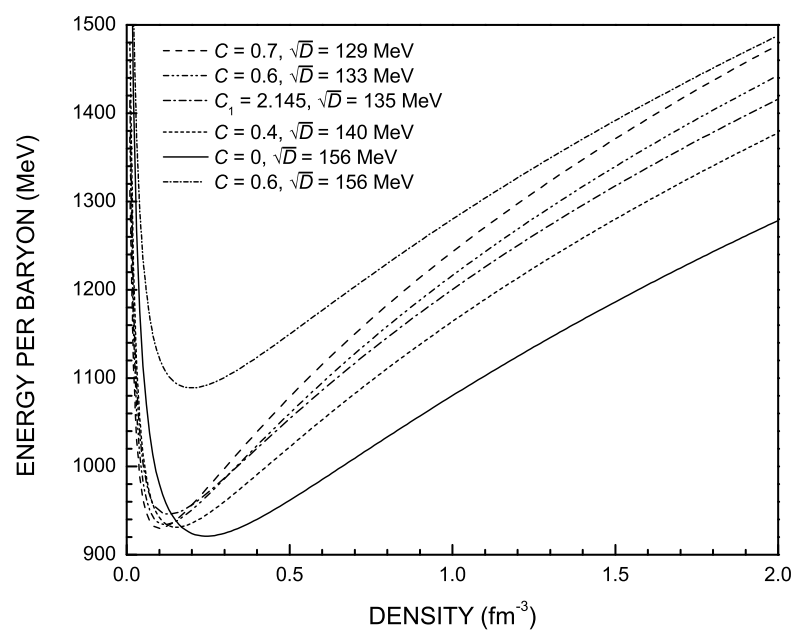

FIG. 5. Energy per baryon as functions of the baryon number density for the mass scaling in Eq. (67) at $m_{u 0}=5 \mathrm{MeV}$, $m_{d 0}=10 \mathrm{MeV}, m_{s 0}=100 \mathrm{MeV}$, with different $D$ and $C$ values indicated in the legend.

According to Eq. (47), the true and effective chemical potentials for each flavor of quarks differ merely by a common quantity $\mu_{\mathrm{I}}$. Thus the effective chemical potentials also satisfy the similar weak equilibrium conditions:

$$
\mu_{u}^{*}+\mu_{e}=\mu_{d}^{*}=\mu_{s}^{*} .
$$

Because electrons do not participate in strong interactions, the corresponding mass is constant. Consequently, the effective and true chemicals potential of electrons are the same.

We can also directly solve for the effective chemical potentials from Eqs. (70)-(72), and then calculate all other thermodynamic quantities from the derived expressions.

To calculate the corresponding thermodynamic quantities such as the true chemical potentials and the pressure etc, we need to provide the derivative of the quark mass with respect to the density. For Eq. (67), it is simply

$$
\frac{\mathrm{d} m_{i}}{\mathrm{~d} n_{\mathrm{b}}}=-\frac{D}{3 n_{\mathrm{b}}^{4 / 3}}+\frac{C}{3 n_{\mathrm{b}}^{2 / 3}}
$$

With the quark mass scaling in Eq. (67) and the corresponding derivative in Eq. (73), we plot the equation of state (EOS) of SQM in Fig. 5 for the parameters $(C, \sqrt{D})$ indicated by solid dots in Fig. 4 From Fig. 5 we have two observations: (1) For parameters within the stable region, the EOS is stiffer than those out of the region; (2) within the region, the stiffness increases with increasing $C$ and decreasing $D$.

For the running coupling case of Eq. (63), we have

$$
\frac{\mathrm{d} m_{i}}{\mathrm{~d} n_{\mathrm{b}}}=-\frac{D}{3 n_{\mathrm{b}}^{4 / 3}}+\frac{C_{1}}{3 n_{\mathrm{b}}^{2 / 3}}\left[a_{1}+\nu \frac{\mathrm{d} a_{1}}{\mathrm{~d} \alpha} \frac{\mathrm{d} \alpha}{\mathrm{d} u} \frac{\mathrm{d} u}{\mathrm{~d} \nu}\right]
$$

The derivative $\mathrm{d} a_{1} / \mathrm{d} \alpha$ can easily be obtained by dif-

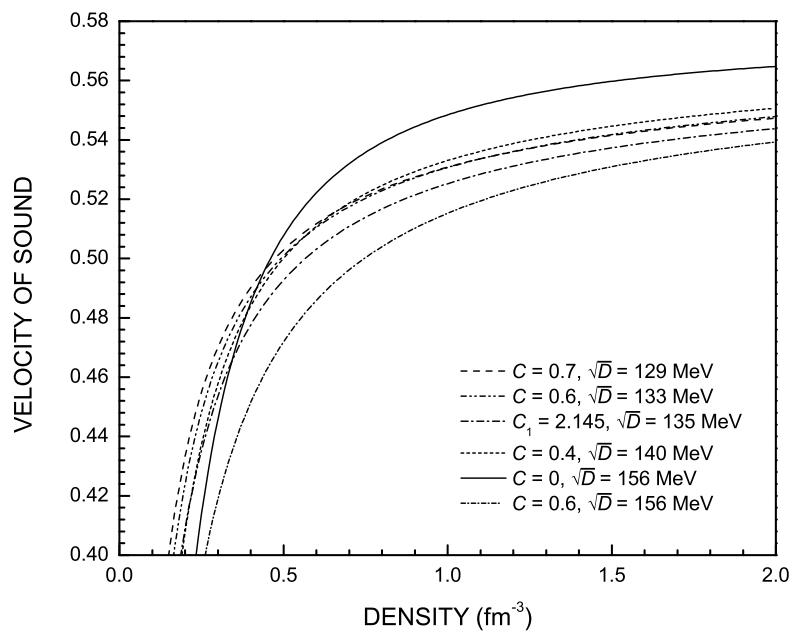

FIG. 6. The velocity of sound in SQM.

ferentiating Eq. (56), giving

$$
\frac{\mathrm{d} a_{1}}{\mathrm{~d} \alpha}=\frac{4(1-2 \alpha)^{-4 / 3}}{3 a_{1}\left(\frac{4+a_{1}^{2}}{\sqrt{1+a_{1}^{2}}}+8 a_{1}^{2} \ln \frac{1+\sqrt{1+a_{1}^{2}}}{a_{1}}\right)} .
$$

Simply with the leading expression in Eq. (62) we have

$$
\frac{\mathrm{d} a_{1}}{\mathrm{~d} \alpha}=\frac{1}{\sqrt{6 \alpha}} .
$$

Because we are trying to include first-order perturbative interaction, we use the leading expression in Eqs. (62) and (76) in the numerical calculations of the present paper.

The derivative $\mathrm{d} \alpha / \mathrm{d} u$ in Eq. (74) should not be replaced with the right-hand side of Eq. (64). Instead, the expression is useable depending on which equation, Eq. (65) or Eq. (66), is used. In the former case we have

$$
\frac{\mathrm{d} \alpha}{\mathrm{d} u}=-\frac{\alpha^{2}}{u}\left[2 \beta_{0}+\frac{\beta_{1}}{\beta_{0} \ln (u / \Lambda)}\right] .
$$

Otherwise, if the latter is used, one then has

$$
\frac{\mathrm{d} \alpha}{\mathrm{d} u}=\frac{1}{\beta_{0} u}\left[\frac{2}{(u / \Lambda-\Lambda / u)^{2}}-\frac{1}{2 \ln ^{2}(u / \Lambda)}\right] .
$$

Finally, we discuss the relation between the holistic Fermi momentum $\nu$ and the renormalization subtraction point $u$. In principle, the exact relation is not available presently. Phenomenologically we also expand it according to the Fermi momentum $\nu$ and take to the first order as

$$
u=c_{0}+c_{1} \nu .
$$

To use Eq. 65), we have to choose a comparatively large value for $c_{0}$ because it should map the $u$ value into 


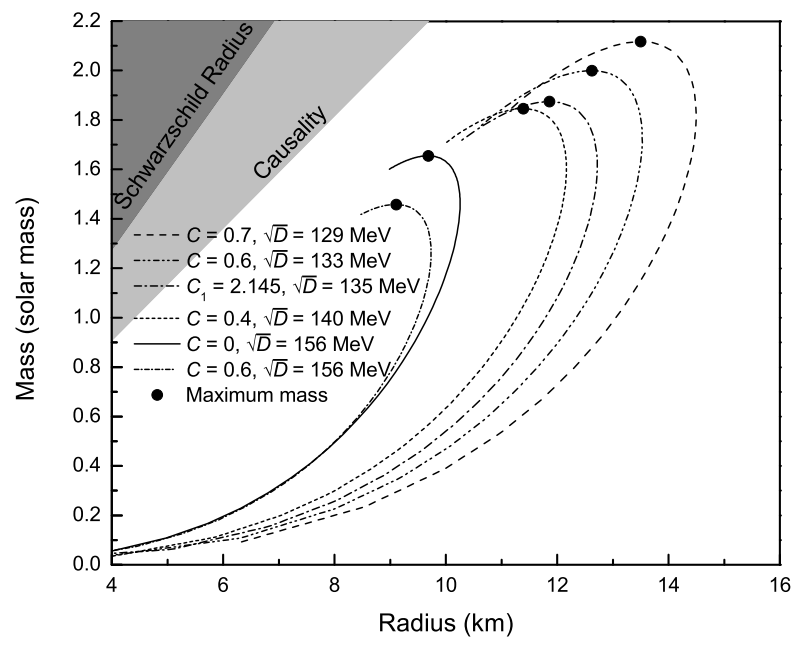

FIG. 7. Mass-radius relation of strange stars for various parameter sets.

a reasonable perturbative range. We take $c_{0}=M_{\mathrm{N}}=$ $938.926 \mathrm{MeV}$. For analytic Eq. (66), however, one does not need to take care of this, and a smaller one, e.g., $c_{0}=\Lambda$, can be taken.

As for the $c_{1}$ value, it is generally between 2 and 3 , and we take a modest value as $c_{1}=2.5$.

In Fig. 5. the dash-dot-dotted curve is for $C_{1}=2.145$ and $\sqrt{D}=135 \mathrm{MeV}$ with the running coupling Eq. (65).

Based on the results in Fig. 5. we can obtain the velocity of sound for SQM using the formula

$$
v=\sqrt{\frac{\mathrm{d} P}{\mathrm{~d} E}} .
$$

with the results given in Fig. 6. As the baryon number density increases, the velocity of sound also increases. At higher densities, the curve corresponding to a larger $C$ value approaches to the ultrarelativistic case $(1 / \sqrt{3})$ more slowly. This is understandable since there are still perturbative interactions at higher densities.

\section{PROPERTIES OF STRANGE STARS}

The quark star has been an interesting subject in nuclear physics, astrophysics, as well as in some other important fields. Pioneer works were done with the earlier version of the bag model [1, 83 85]. Many further investigations have appeared in the past two decades, such as in Refs. [86, 87]. Models other than the bag one have also been applied, e.g. the $\mathrm{SU}(3)$ parity doublet model 88, the NJL [89], the Komathiraj-Mahara method [90, 91], etc.

Recently, the stellar properties were studied with the reciprocity scaling in Eq. (1) and the thermodynamic treatment TD-2 [58]. It is found that the maximum mass exceeds $2 M_{\odot}$ for all the model parameters in the whole stability window. Therefore, this model can describe massive quark stars, but fails to accommodate stars with low radii. As also noted by the authors, the treatment suffers from thermodynamic inconsistency.

With the consistent thermodynamic treatment TD-3, the mass-radius relation was previously calculated with the cubic-root scaling [46]. It was found that the maximum mass is normally much smaller than $2 M_{\odot}$ [46]. Using TD-3 with the reciprocity, the case is also similar, though it can describe stars with low radii [16]. Even when one changes the confinement exponent, which is unity in Eq. (1) and a third in Eq. (2), to other values, or considering isospin interactions [57], the case is still similar 92].

In the preceding sections, we have obtained a new quark mass scaling which includes both the confinement and the leading-order perturbative interactions. On application of the scaling and the fully consistent thermodynamic treatment, we obtain the new EOS in Fig. 5. Let us now apply it to solve the Tolman-OppenheimerVolkov equation

$$
\frac{\mathrm{d} P}{\mathrm{~d} r}=-\frac{G m E}{r^{2}} \frac{(1+P / E)\left(1+4 \pi r^{3} P / m\right)}{1-2 G m / r}
$$

with the subsidiary condition

$$
m=\int_{0}^{r} 4 \pi E r^{2} \mathrm{~d} r
$$

For a concise description of the solving process, one can refer to Ref. [46]. The results are given in Fig. 7 where the maximum mass is marked with full dots. Obviously the maximum mass can be as large as $2 M_{\odot}$.

Generally, the maximum star mass increases with increasing perturbative strength parameter $C$, but decreases with increasing the confinement strength parameter $D$. Therefore, going toward the lower-right direction of the stability region (shaded in Fig. 4) increases to as large as $2 M_{\odot}$, while going in the upper-right direction, it decreases to give stars with small radii, and the maximum mass is also small, much less than $2 M_{\odot}$.

It should be noted that when a comparatively large $C$ value is used to produce a large maximum mass, the density at the surface of the star becomes very small, even below the normal nuclear saturation density. To show this explicitly, we draw the density profiles as a function of the radius with four sets of the parameter pair $(C, \sqrt{D})$. The panel (a) is for the parameter pair $(C, \sqrt{D})=(0,156 \mathrm{MeV})$. In this case, the maximum star mass is smaller $\left(\sim 1.6 M_{\odot}\right)$, and the surface density is comparatively higher $\left(0.24 \mathrm{fm}^{-3}\right)$. The panels $(\mathrm{b}),(\mathrm{c})$, and (d) are, respectively, for $(0.4,140 \mathrm{MeV}),(0.6,135$ $\mathrm{MeV}),(0.7,129 \mathrm{MeV})$. In these cases, the maximum star mass becomes larger, but the surface density gets low, even lower than the normal nuclear matter one. This is clearly a signal for the phase transition to nuclear matter. Therefore, further investigations on the QCD phase diagram [2] are necessary in future works with the new quark mass scaling. 

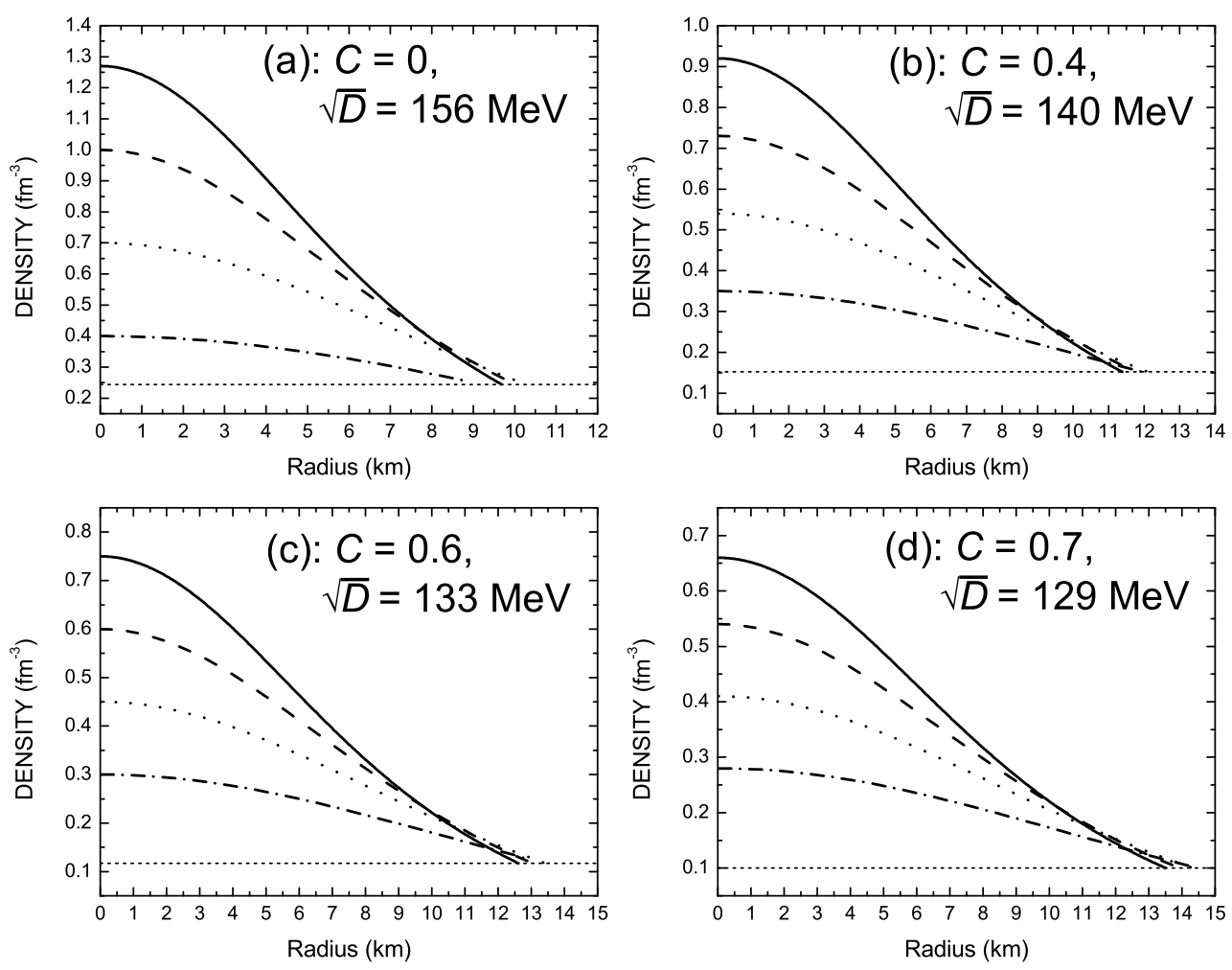

FIG. 8. Density profiles for different values of the parameters $C$ and $\sqrt{D}$. The uppermost curve on each panel is for the largest acceptable central density, while the horizontal line corresponds to the surface density of the star.

Now we discuss a little about how to ensure the asymptotic freedom at extremely high density. As one can see, the perturbative term in the new quark mass scaling does not obviously decrease with increasing density. This problem is not presently serious because, on one hand, the decreasingly running factor $C$ ensures that the increasing velocity is slow, and on the other hand, quarks become asymptotically free rather slowly 93]. Also, if necessary, one can use a damping form factor, to obviously ensure it, as done in Refs. [56, 57] for considering the isospin or one-gluon-exchange interactions.

Furthermore, the color superconductivity [67] and the strong magnetic field [94, 95], which possibly play important roles at extremely high density, have not been considered. The influence of these factors to the EOS of SQM, and accordingly to the structure of compact stars, should be considered in future papers.

\section{SUMMARY}

We have clarified the inconsistency issues in the previous thermodynamic treatments on SQM with density and/or temperature dependent particle masses. We find that the fundamental differential equation of standard thermodynamics dose not need to be modified. Instead, the previous treatments with nonzero pressure at the energy minimum should be discarded, while calculations in
TD-3 are still correct, and for a full thermodynamic consistency, one just needs to regard the original chemical potentials as effective ones.

By expanding the equivalent mass to a Laurant series and taking the leading terms in both directions, we arrive at a new quark mass scaling with linear confinement and leading-order perturbative interactions. With the new quark mass scaling and the present thermodynamics treatment, we have studied the EOS of SQM with both constant and running strong coupling. It is found that the new model, which can be called an equiparticle model, gives the EOS which can describe massive quark stars with gravitation mass as large as 2 times the solar mass. At the same time, it can also describe stars with low radii, depending on the comparative strength of the confinement and leading perturbative interactions.

\section{Appendix A: Necessary conditions for the fully consistent thermodynamics of strange quark matter}

Strange quark matter is usually composed of up $u$, down $d$, and strange $s$ quarks and electrons $(e)$. Due to weak reactions, the chemical potentials $\mu_{i}(i=u, d, s, e)$ satisfy the weak equilibrium condition, the charge neutrality, and the baryon number density conservation, as in Eqs. (69)-(71). To solve out the chemical potentials $\mu_{i}(i=u, d, s, e)$ from these equations for a given baryon 
number density $n$, one should know the relation between the chemical potentials $\mu_{i}$ and particle number densities $n_{i}$. Furthermore, one should know how to calculate the system energy density (the free energy at finite temperature), the pressure, and other thermodynamic quantities that belong to thermodynamic treatments. Here we derive model-independent relations any fully consistent thermodynamic treatment for SQM must satisfy.

Let us start from the fundamental thermodynamic differential equation by using the free energy $\bar{F}=\bar{E}-T \bar{S}$ as

$$
\mathrm{d} \bar{F}=-\bar{S} \mathrm{~d} T-P \mathrm{~d} V+\sum_{i} \mu_{i} \mathrm{~d} \bar{N}_{i} .
$$

For an isotropic system with homogeneously distributed particles, it is convenient to define the corresponding intensive quantities: the energy density $E \equiv$ $\bar{E} / V$, the free-energy density $F \equiv \bar{F} / V$, the entropy density $S \equiv \bar{S} / V$, and the particle number densities $n_{i} \equiv \bar{N}_{i} / V$. Equation (A1) then becomes

$$
\begin{aligned}
\mathrm{d} F= & -S \mathrm{~d} T+\sum_{i} \mu_{i} \mathrm{~d} n_{i} \\
& +\left(-P-F+\sum_{i} \mu_{i} n_{i}\right) \frac{\mathrm{d} V}{V}
\end{aligned}
$$

Equation A2 indicates that one should use $T,\left\{n_{i}\right\}$, and $V$ as the independent state variables if the free-energy density $F$ is chosen as the characteristic quantity. If $F$ as a function of the independent state variables is known, all other thermodynamic quantities can be calculated from it by

$$
\begin{aligned}
S & =\left.\frac{\mathrm{d} F}{\mathrm{~d} T}\right|_{\left\{n_{k}\right\}}, \\
\mu_{i} & =\left.\frac{\mathrm{d} F}{\mathrm{~d} n_{i}}\right|_{T,\left\{n_{k \neq i}\right\}}, \\
P & =-F+\sum_{i} \mu_{i} n_{i} .
\end{aligned}
$$

In general e.g. for strangelets, $F$ is a function of $T$, $\left\{n_{i}\right\}$, and $V$. In this case, a term like $V \partial F / \partial V$ should be added to the right-hand side of Eq. A5. In the present case, however, we are considering strange quark matter whose finite size effect is not significant. The free-energy density $F$ does not depend on the volume, and Eq. A2 accordingly becomes

$$
\mathrm{d} F=-S \mathrm{~d} T+\sum_{i} \mu_{i} \mathrm{~d} n_{i}
$$

Because all the second-order mixed partial derivatives of an arbitrary analytic function are equal to each other mathematically, we can easily obtain, from Eqs. (A3) and (A4), the relations

$$
\left.\Delta_{i} \equiv \frac{\mathrm{d} S}{\mathrm{~d} n_{i}}\right|_{T,\left\{n_{k \neq i}\right\}}-\left.\frac{\mathrm{d} \mu_{i}}{\mathrm{~d} T}\right|_{T,\left\{n_{k}\right\}}=0
$$

and

$$
\left.\Delta_{i j} \equiv \frac{\mathrm{d} \mu_{i}}{\mathrm{~d} n_{j}}\right|_{T,\left\{n_{k \neq j}\right\}}-\left.\frac{\mathrm{d} \mu_{j}}{\mathrm{~d} n_{i}}\right|_{T,\left\{n_{k \neq i}\right\}}=0,
$$

where $i, j=u, d, s$ quarks. Equations (A7) and A8 are nothing but the Cauchy conditions for the right-hand side of Eq. A6 to be integrable when the free energy is chosen as the characteristic function.

When additional conditions are provided, e.g. these in Eqs. (69)-(71), the free-energy density $F$ is determined as a function of the temperature and the density $n \equiv$ $\sum_{q} n_{q} / 3$. At a given $T$, Eq. (A6) gives

$$
\frac{\mathrm{d} F}{\mathrm{~d} n}=\sum_{i} \mu_{i} \frac{\mathrm{d} n_{i}}{\mathrm{~d} n}
$$

Therefore, we have

$$
\begin{aligned}
n \frac{\mathrm{d} F}{\mathrm{~d} n} & =n\left[\sum_{q} \mu_{q} \frac{\mathrm{d} n_{q}}{\mathrm{~d} n}+\mu_{e} \frac{\mathrm{d} n_{e}}{\mathrm{~d} n}\right] \\
& =n\left[\mu \sum_{q} \frac{\mathrm{d} n_{q}}{\mathrm{~d} n}-\mu_{e} \frac{\mathrm{d} n_{u}}{\mathrm{~d} n}+\mu_{e} \frac{\mathrm{d} n_{e}}{\mathrm{~d} n}\right] \\
& =n\left[\mu \frac{\mathrm{d}}{\mathrm{d} n}\left(\sum_{q} n_{q}\right)-\mu_{e} \frac{\mathrm{d}}{\mathrm{d} n}\left(n_{u}-n_{e}\right)\right] \\
& =n\left(3 \mu-\mu_{e}\right)=\mu \sum_{q} n_{q}-\mu_{e} n \\
& =\sum_{q} \mu_{q} n_{q}+\mu_{e} n_{u}-\mu_{e} n \\
& =\sum_{i} \mu_{i} n_{i}-\mu_{e} n_{e}+\mu_{e} n_{u}-\mu_{e} n \\
& =\sum_{i} \mu_{i} n_{i}+\mu_{e}\left(n_{u}-n_{e}-n\right) \\
& =\sum_{i} \mu_{i} n_{i},
\end{aligned}
$$

where the summation on the index $i$ goes over $u, d, s, e$, while that on $q$ goes over $u, d, s$. We have used the chemical equilibrium $\mu_{u}+\mu_{e}=\mu_{d}=\mu_{s} \equiv \mu$ and the relation $n_{u}-n_{e}=n$ obtained by Eq. (70) plus Eq. (71). Considering Eq. (A5), we thus have

$$
P=n^{2} \frac{\mathrm{d}}{\mathrm{d} n}\left(\frac{F}{n}\right)_{T} .
$$

i.e.,

$$
\Delta \equiv P-n^{2} \frac{\mathrm{d}}{\mathrm{d} n}\left(\frac{F}{n}\right)_{T}=0 .
$$

Equation (A12) means that the pressure at the freeenergy minimum (the energy minimum at zero temperature) is exactly zero. In other words, the (free-)energy minimum is a mechanically stable state. Equations A7 and (A8) are the Cauchy conditions which ensure the 
existence of the system. Therefore, Eqs. (A7), A8, and (A12) are the necessary conditions for any consistent thermodynamics of strange quark matter. One can use the pressure, (free-)energy density, chemical potentials, and entropy given by a phenomenological model to calculate the $\Delta$ 's defined in Eqs. (A7), (A8), and (A12). Any obvious difference from zero indicates the inconsistence

[1] E. Witten, Phys. Rev. D 30, 272 (1984).

[2] G. X. Peng, U. Lombardo, and A. Li, Phys. Rev. C 77, 065807 (2008).

[3] X. J. Wen, Physica A 392, 4388 (2013).

[4] X. P. Zheng, X. Zhou, and S. H. Yang, Phys. Lett. B 729, 79 (2014).

[5] G. F. Burgio, M. Baldo, P. K. Sahu, and H. J. Schulze, Phys. Rev. C 66, 025802 (2002).

[6] D. Logoteta and I. Bombarci, Phys, Rev. D 88, 063001 (2013).

[7] M. Orsaria, H. Rodrigues, F. Weber, and G. A. Contrera, Phys. Rev. C 89, 015806 (2014).

[8] U. A. Wiedemann, Nucl. Phys. A 904-905, 3c (2013).

[9] I. Bombarci, G. Lugones, and I. Vidana, Astron. Astrophy. 462, 1017 (2007).

[10] E. Farhi and R. L. Jaffe, Phys. Rev. D 30, 2379 (1984).

[11] G. X. Peng, X. J. Wen, and Y. D. Chen, Phys. Lett. B 633, $314(2006)$.

[12] S. B. Shaulov and S. P. Bezshapov, EPJ Web Conf. 52, 04010 (2013).

[13] E. Finch, J. Phys. G 32, S251 (2006).

[14] B. Monreal, J. High Energy Phys. 02 (2007) 077.

[15] M. Angeles Perez-Garcia, J. Silk, and J. R. Stone, Phys. Rev. Lett. 105, 141101 (2010).

[16] V. Dexheimer, J. R. Torres, and D. P. Menezes, Eur. Phys. J. C 73, 2569 (2013).

[17] M. Bagchi, S. Ray, M. Dey, and J. Dey, Astron. Astrophys. 450, 431 (2006).

[18] M. Sinha, X. G. Huang, and A. Sedrakian, Phys. Rev. D 88, 025008 (2013).

[19] Y. Nambu and G. Jona-Lasino, Phys. Rev. 122, 345 (1961); ibid. 124, 246; M. G. Paoli, and D. P. Menezes, Eur. Phys. J. A 46, 413 (2010).

[20] E. S. Fraga, R. D. Pisarski, J. Schaffner-Bielich, Phys. Rev. D 63, 121702(R) (2001); G. X. Peng, Europhys. Lett. 72,69 (2005).

[21] S. Plumari, G. F. Bugio, and V. Greco, Phys. Rev. D 88, 083005 (2013).

[22] R. X. Xu, Astrophys. J., 596 L59 (2003); Int. J. Mod. Phys. D 19, 1437 (2010).

[23] G. S. Khadekar and R. Wanjari, Int. J. Theor. Phys. 51, 1408 (2012).

[24] P. K. Sahoo and B. Mishra, Int. J. Pure Appl. Math. 82 (1), 87 (2013).

[25] X. J. Wen, Phys. Rev. D 88, 034031 (2013).

[26] A. A. Isayev and J. Yang, J.Phys. G 40, 035105 (2013).

[27] X. Y. Wang and I. A. Shovkovy, Phys. Rev. D 82, 085007 (2010); M. Huang and I. A. Shovkovy, Phys. Rev. D 70, 051501(R) (2004). degree of the corresponding thermodynamic treatment.

\section{ACKNOWLEDGMENTS}

The authors would like to thank the support from National Natural Science Foundation of China (No. 11135011) and the key project from Chinese Academy of Sciences (12A0A0012).

[28] C. Wu, W. L. Qian, and R. K. Su, Phys. Rev. C 72, 035205 (2005); T. Bao, G. Z. Liu, E. G. Zhao, and M. F. Zhu, Eur. Phys. J A 38, 287 (2008).

[29] A. Chodos, R. L. Jaffe, K. Johnson, C. R. Thorn, and V. F. Weisskopf, Phys. Rev. D 9, 3471 (1974).

[30] J. Madsen, Phys. Rev. Lett. 85, 4687 (2000).

[31] F. Weber, O. Hamil, K. Mimura, and R. Negreiros, Int. J. Mod. Phys. D 19, 1427 (2010).

[32] H. Rodrigues, F. Weber, and S. B. Duarte, Astrophys. Space Sci. 344, 229 (2013).

[33] Proceedings of the International Conference on Strangeness in Quark Matter (SQM 2011), edited by H. Bialkowska and W. Florkowski [Acta Phys. Pol. B 43, 497 (2013)].

[34] V. Goloviznin and H. Satz, Z. Phys. C 57, 671 (1993).

[35] M. I. Gorenstein and S. N. Yang, Phys. Rev. D 52, 5205 (1995).

[36] K. Schertler, C. Greiner, and M. H. Thoma, Nucl. Phys. A 616, 659 (1997).

[37] A. Peshier, B. KK̈ampfer, and G. Soff, Phys. Rev. C 61, 045203 (2000).

[38] V. Bannur, Phys. Rev. C 75, 044905 (2007).

[39] F. G. Gardim and F.M. Steffens, Nucl. Phys. A 825, 222 (2009).

[40] J. Cao, Y. Jiang, W. M. Sun, and H. S. Zong, Phys. Lett. B 711, 65 (2012).

[41] L. J. Luo, J. C. Yan, W. M. Sun, and H. S. Zong, Eur. Phys. J. C 73, 2626 (2013).

[42] G. N. Fowler, S. Raha, and R. M. Weiner, Z. Phys. C 9, 271 (1981).

[43] M. Plümer, S. Raha, and R. M. Weiner, Phys. Lett. B 139, 198 (1984).

[44] S. Chakrabarty, S. Raha, and B. Sinha, Phys. Lett. B 229, 112 (1989).

[45] O. G. Benvenuto and G. Lugones, Phys. Rev. D 51, 1989 (1995); G. Lugones and O. G. Bevenuto, Phys. Rev. D 51, 1276 (1995).

[46] G. X. Peng, H. C. Chiang, B. S. Zou, P. Z. Ning, and S. J. Luo, Phys. Rev. C 62, 025801 (2000).

[47] X. J. Wen, X. H. Zhong, G. X. Peng, P. N. Shen, and P. Z. Ning, Phys. Rev. C 72, 015204 (2005).

[48] S. Y. Yin and R. K. Su, Phys. Rev. C 77, 055204 (2008).

[49] P. Wang, Phys. Rev. C 62, 015204 (2000).

[50] G. X. Peng, H. C. Chiang, J. J. Yang, L. Li, and B. Liu, Phys. Rev. C 61, 015201 (1999).

[51] Y. Zhang, R. K. Su, S. Q. Ying, and P. Wang, Europhys. Lett. 56, 361 (2001); Y. Zhang and R. K. Su, Phys. Rev. C 65, 035202 (2002). 
[52] X. P. Zheng, X. W. Liu, M. Kang, and S. Yang, Phys. Rev. C 70, 015803 (2004).

[53] G. Lugones and J. E. Horvath, Int. J. Mod. Phys. D, 12, 495 (2003).

[54] A. Li, G. X. Peng, and J. F. Lu, Res. Astron. Astrophys. 11, 482 (2011).

[55] M. Modarres and H. Gholizade, Int. J. Mod. Phys. E 17, 1335 (2008).

[56] S. W. Chen and G. X. Peng, Commun. Theor. Phys. 57, 1037 (2012); Chin. Phys. C 36, 947 (2012).

[57] P. Z. Chu and L. W. Chen, Astrophys. J. 780, 135 (2013).

[58] J. R. Torres and D. P. Menezes, Europhys. Lett. 101, 42003 (2013).

[59] J. Antoniadis, P. C. C. Freire, N. Wex et al, Science 340, 1233232 (2013).

[60] P. B. Demorest, T. Pennucci, S. M. Ransom et al, Nature(London) 467, 1081 (2010).

[61] J. D. Walecka, Theoretical Nuclear and Subnuclear Physics (Oxford University, New York, 1995).

[62] E. M. Henley, and H. Mther, Nucl. Phys. A 513, 667 (1990).

[63] P. Marmier, and E. Sheldon, Physics of Nuclei and Particles (Academic Press, New York, 1970), p. 1406.

[64] B. D. Serot and J. D. Walecka, Int. J. Mod. Phys. E 6, 515 (1997); Adv. Nucl. Phys. 16, 1 (1986).

[65] X. J. Wen, J. Y. Li, J. Q. Liang, and G. X. Peng, Phys. Rev. C 82, 025809 (2010).

[66] G. X. Peng, H. C. Chiang, and P. Z. Ning, Int. J. Mod. Phys. A 18, 3151 (2003).

[67] K. Rajagopal and F. Wilczek, arXiv:hep-ph/0011333

[68] M. G. Alford, Annu. Rev. Nucl. Part. Sci. 51, 131 (2001).

[69] M. Alford, K. Rajagopal, S. Reddy, and F. Wilczeck, Phys. Rev. D 64, 074017 (2001).

[70] G. Lugones and J. E. Horvath, Phys. Rev. D 66, 074017 (2002).

[71] Y. D. Chen, G. X. Peng, and X. J. Wen, High Energy Phys. Nucl. Phys. 31, 269 (2007); Q. Chang, S. W. Chen, and G. X. Peng, Sci. China Phys. Mech. Astron. 56, 1730 (2013).

[72] X. J. Wen, G. X. Peng, and P. N. Shen, Int. J. Mod. Phys. A 22, 1649 (2007).

[73] G. X. Peng, Nucl. Phys. A 747, 75 (2005).
[74] R. Baier and K. Redlich, Phys. Rev. Lett. 84, 2100 (2000).

[75] B. A. Freedman and L. D. McLerran, Phys. Rev. D 16, 1169 (1978).

[76] V. Baluni, Phys. Rev. D 17, 2092 (1978).

[77] T. Toimela, Int. J. Theor. Phys. 24, 901 (1985).

[78] G. 't Hooft, Nucl. Phys. B 61, 455 (1973); W. A. Bardeen, A. J. Buras, D. W. Duke, and T. Muta, Phys. Rev. D 18, 3998 (1978).

[79] T. van Ritbergen, J. A. M. Vermaseren, and S. A. Larin, Phys. Lett. B 400379 (1997).

[80] G. X. Peng, Phys. Lett. B 634, 413 (2006).

[81] D. V. Shirkov and I. L. Solovtsov, Phys. Rev. Lett. 79, 1209 (1997); arXiv:hep-ph/9604363.

[82] G. X. Peng, U. Lombardo, M. Loewe, and H. C. Chiang, Phys. Lett. B 548, 189 (2002).

[83] N. Itoh, Prog. Theor. Phys. 44, 291 (1970).

[84] C. Alcock, E. Farhi, and A. Olinto, Astrophys. J. 310, 261 (1986).

[85] P. Haensel, J. L. Zdunik, and R. Schaeffer, Astron. Astrophys. 160, 121 (1986).

[86] M. Alford, D. Blaaschke, A. Drago, et al, Nature (London) 445, E7 (2007); M. Alford, M. Braby, M. Paris, and S.Reddy, Astrophys. J. 629, 969 (2005).

[87] S. Weissenborn, I. Sagert, G. Pagliara, M. Henpel, and J. Schaffner-Bielich, Atrophys. J. 740, L14 (2011).

[88] V. Dexheimer, J. Steinheimer, R. Negreiros, and S. Schramm, Phys. Rev. C, 87, 015804 (2013).

[89] T. Klähn, R. Lastowiecki, and D. Blaschke, Phys. Rev. D 88, 085001 (2013).

[90] K. Komathiraj and S.D. Maharaj, Int. J. Mod. Phys. D 16, 1803 (2007).

[91] D. Maharaj, J. M. Sunzu, and S. Ray, Eur. Phys. J. Plus 129, 3 (2014)

[92] A. Li, R. X. Xu, and J. F. Lu, Mon. Not. R. Astron. Soc. 402, 2715 (2010).

[93] T. D. Lee, Nucl. Phys. A 750, 1 (2005).

[94] S. Chakrabarty, Phys. Rev. D 54, 1306 (1996).

[95] X. J. Wen, S. Z. Su, D. H. Yang, and G.X. Peng, Phys. Rev. D 86, 034006 (2012). 